\title{
Large-eddy Simulation of a Large-scale Methane Pool Fire
}

\author{
S. FERRARIS, J.X. WEN, and S. DEMBELE \\ School of Engineering, Kingston University \\ Friars Avenue \\ Roehampton Vale, London, SW15 3DW, UK
}

\begin{abstract}
In this study a modified version of the Conditional Source Estimation (CSE) model has been developed. It is assessed on a diffusion flame (SANDIA Flame D) and a $1 \mathrm{~m}$ diameter methane pool fire. In both cases it has been found that mean mass species concentration, temperature, scalar and velocity fields agree reasonably well with the experiments. The main goal of this paper is to prove the possibility of employing the CSE as a model of low computational cost for large fire simulations. The work also highlights the importance of soot and radiation models in large scale fires.
\end{abstract}

KEYWORDS: large eddy simulation (LES), conditional source estimation (CSE), pool fire, SANDIA D flame

\section{INTRODUCTION}

Large-Eddy Simulations (LES) resolves the major turbulent scales and models the influence of small-scale eddies at sub-grid level. This latter characteristic appears to be suitable for large-scale fires where the mixing process is mainly dominated by large eddies. This is particular interesting for simulations of turbulent reactants flows.

Nevertheless, the combustion takes place at the smallest scales of this spectrum therefore it must be modeled completely. Among the most difficult combustion models are the direct method, the transported probability function (pdf) method, the conserved scalar method, etc.

The pdf concept has been used in conserved scalar methods, which assumes that the chemical state and therefore the specie concentrations are related with a conserved scalar, usually the mixture fraction $(Z)$. Then, by presuming the sub-grid scale pdf of the mixture fraction $P(Z)$, the filtered species mass fractions $Y_{I}$ in each computational cell can be evaluated by:

$\overline{Y_{i}}(\bar{x})=\int_{0}^{1} Y \mid Z(\bar{x}) P\left(\bar{x}, z^{22}, Z^{*}\right) d Z^{*}$

$\mathrm{x}$ is the position vector, $P(Z)$ is usually assumed to follow a Beta distribution, parameterised with two moments, the filtered mixture fraction and its variance. The first moment is determined by solving the transport equation for $Z$ and the variance can be either calculated by a sub-grid model or solved through another transport equation.

There are many sub-grid models for mixture fraction variance. In the present study the scale similarity model proposed in [1] is adopted. The constant coefficient was dynamically calculated using [2]. 
Different approaches have been used to specify the function $Y(Z)$ in Eq. 1. Among them are the infinitely fast chemistry assumptions [2], the equilibrium assumption [1], the conditional moment closure method (CMC) [3], the conditional source estimation (CSE) [4], the steady laminar flamelet model (LFM) [5] and the unsteady laminar flamelet model (ULFM) [6].

A balance should be found between accuracy and computational cost. From the accuracy point of view, the ULFM [6] seems to be the most promising since it considers the evolution in time of the flamelets, whereas the CSE version used here considers steady flamelet of the ULFM that yields excellent results in [6].

From the computational point of view, CSE only solves the chemical reactions independently of the actual flow calculations and store them in a look-up table. Two inversions are necessary on each plane to obtain the coefficients while in ULFM the chemical reaction chain is solved on each plane.

The CSE approach is adopted in the present study because of its relatively low computational cost and reasonable accuracy that make it suitable for modeling large-scale pool fire. The present authors have developed a modified version of the original CSE method proposed by Bushe \& Steiner [4]. This modified approach is applied to LES for a turbulent diffusion flame [7] as a benchmark scenario and to a one meter diameter methane pool fire [8].

\section{MATHEMATICAL MODEL}

\section{Large Eddy Simulation (LES)}

In LES equations are obtained applying a spatial density-weight filter to the energy, continuity, momentum and conserved scalar equations. The sub-grid scale stress in the momentum equation needs a closure. Since the small scales tend to be more isotropic than the large ones, it should be possible to parameterise them using simple models. Most sub-grid-scale stress models (SGS) are based on an eddy viscosity assumption. In the most commonly used model, developed by Smagorinsky, the eddy viscosity $\mu_{t}$ is obtained by assuming that the small scales are in equilibrium, so the energy production and dissipation are in balance. In the present work the Smagorinsky constant is Cs $=0.12$ and the turbulent Schmidt number is considered to be constant $S c_{t}=0.4$.

\section{The CMC Approach}

As mentioned above there are many approaches to establish the function of the conditional specie mass concentrations $\left(Y_{I}\right)$ and temperature $(T)$. CMC approach solves a set of equations to compute $\left.Y_{I}\right|_{Z}$ and $\left.T\right|_{Z}$. ULMF solves the unsteady flamelet equations coupled with the flow solver to obtain the conditional quantities [6]. Alternatively, CSE proposes to estimate the conditional variables performing the inversion of Eq. 1 to obtain $\left.Y_{I}\right|_{Z}$ and $\left.T\right|_{Z}$. CSE was firstly proposed by Bushe \& Steiner [3].

CSE assumes that the conditional quantities are similar in every flow cell belonging to planes equidistant to the nozzle. The later assumption was extensively confirmed by experiments [9] for certain types of flows.

However, the inversion of Eq. 1 performed by simple methods has been shown to be very unstable and sometimes physically meaningless. This instability is inherent to the inversion process itself [10]. 
To overcome this difficulty another technique called decomposition was proposed by Grout and Bushe [11]. In the decomposition method, a set of basis functions is chosen and $\left.T\right|_{Z}$ is decomposed in the following way:

$T \mid Z=\sum_{i=1}^{n} a_{i} \theta_{x i}(Z)$

where $a_{i}$ are the coefficients of the decomposition. $\theta_{x i}$ are the base functions of the temperature, $x i$ denotes the dissipation rate which this function is related to, $n$ is the number of dissipation rates considered in the basis ( $n$ will be the number of columns in the basis). Considering Eq. 1 and Eq. 2 and minimizing to find the optimum solution yields,

$\left(K^{T} K+\lambda I\right) a=K^{T} b$

where $K$ is the kernel of the inversion, $I$ is the identity matrix, $\lambda$ is the regularization parameter chosen to add just enough a priori information and to stabilize the inversion, $a$ is the unknown coefficients vector and $b$ is the filtered variable. It is important to highlight that if $\lambda=0$ we have an inversion in a least square sense.

Many base functions could be chosen to perform the inversion, i.e., polynomials, Legendre polynomials, etc. An a-priori selection could be the Laminar Flamelet Functions. This base has the advantage of being actual realizations of the flame in the flow. In order to solve the flamelets, a set of equation must be solved, the so-called unsteady flamelet equations [12]. In the present study the flamelet equations have been solved using the FlameMaster code [13], with 28 species, 72 reactions [14] and considering optical thin gases as radiation loses $\left(\mathrm{CO}_{2}\right.$ and $\left.\mathrm{H}_{2} \mathrm{O}\right)$. In the present case the steady flamelet were solved for dissipation rates ranging from the pure mixing state to the onset of the dissipation.

In the decomposition method the coefficients of the vector $a$ might be used to obtain the conditional species and temperature. This methodology was originally proposed by Bushe and Steiner [4].

From the point of view of the inversion convergence the basis should be as orthogonal as possible in order to pose correctly the inversion problem and thus to avoid instabilities [15].

Nevertheless, the flamelet solution rank is not large (the singular values drop to zero quickly for high frequencies) therefore the basis is not very orthogonal and consequently the inversion is negatively affected. Hence, the computation of the conditional quantities from the temperature field alone produces an ill-posed inversion system. To overcome this it has been proposed to consider another extra scalar quantity to perform the inversion [11], i.e., $\mathrm{CO}_{2}$. Grout and Bushe [11] used an inversion technique called the restrained method, which takes the previous time step solution as departing point for the new solution. In this case the function of $\lambda$ is twofold to stabilise the inversion and to allow the development towards quasi-steady state. 
In the present work an alternative approach is presented. The present method will reduce the number of base functions. This is expected to avoid the instability inherent to the inversion.

In order to reduce the basis various approaches are possible. One option is to reduce the number of temperature base functions through the application of Singular Value Decomposition (SVD) and select those vector with singular values larger than a specific value. This will improve considerably the inversion convergence. Nevertheless, it has the disadvantage that the functions used in the inversion will remain unchanged throughout the flow. Another possible option, which is considered here, is to take the conditional dissipation rate on a plane as an external parameter to select the basis functions for the inversion on the same plane. We perform an inversion to obtain the conditional dissipation rate on planes equidistant to the nozzle departing from local dissipation values. Thereafter, we obtain the maximum conditional dissipation rate $\left(X^{\operatorname{MAX}} \mid z\right)$ on the plane. The basis considered for the inversion are those corresponding to this value of dissipation rate, centered at $X{ }^{M A X} \mid z$ plus a window of $(\Delta X \mid z)$, which it is necessary to determine. Hence, the functions considered are $\theta_{X \max |z+\Delta X| z}$. For the calculation of the dissipation rate we follow [16].

\section{RESULTS}

\section{Jet Diffusion Flame}

\section{Validation Scenario}

The scenario used for the validation of the proposed models is a piloted methane/air jet diffusion flame (SANDIA Flame D) [7] and [17]. The fuel is a $25 / 75 \%$ methane/air mixture. The fuel has been premixed with air in order to minimize the formation of polycyclic aromatic hydrocarbons and soot. The fuel nozzle has a diameter of $D=7.2 \mathrm{~mm}$ and is enclosed by a broad pilot nozzle with a diameter of $D p=18.34 \mathrm{~mm}$. In the experiment the pilot composition and temperature have been adjusted such that the pilot stream has the same equilibrium composition as a mixture fraction of $Z=0.27$ at the copilot and $Z=1$ at the fuel nozzle. The stoichiometric mixture fraction is $Z=0.35$. The fuel bulk velocity is $49 \mathrm{~m} / \mathrm{s}$, which is a Reynolds number of 22400 based on the nozzle diameter. The maximum velocity at the center of the fuel nozzle is $\operatorname{Vmax}=62 \mathrm{~m} / \mathrm{s}$. The flame has been experimentally investigated in [7] and [17] where average and variance value for temperature and species are provided.

\section{Numerical Scheme}

The equations are solved by an explicit second order method both in time and in space. A central difference predictor-corrector scheme is used. The equations are compressive and the advection term is treated in its rotation form. A direct Poisson solver is used for solving the mass conservation equation. The radiation losses are not considered here since this flame is not expected to be very sooty. The flamelet were solved for 25 different values of dissipation rates. The minimum value being $10 \mathrm{~s}^{-1}$ and the maximum, close to extinction, $300 \mathrm{~s}^{-1}$ The species boundary condition are $Y_{\mathrm{CH} 4}=0.15, Y_{\mathrm{O} 2}=0.19$ and $T=300 \mathrm{~K}$ for $Z=1$ and $Y_{\mathrm{CH}_{4}}=0 ., Y_{\mathrm{O} 2}=0.24$ and $T=300 \mathrm{~K}$ for $Z=0$. The discretization used in the mixture fraction space is 35 nodes equally spaced. Rectangular cells are used. The circular nozzle was approximated with $(4 \times 4)$ cells. The low cell 
density in this region can affect the turbulent development near the nozzle but it is not expected to influence the flow further downstream, where it becomes self-similar. The mesh used is $(100 \times 100 \times 250)$ and the domain is $(0.2 \times 0.2 \times 0.55) \mathrm{m}$ in $X, Y$ and $Z$ directions respectively.

\section{Results for SANDIA flame}

Figures 1 and 2 depict the mean and fluctuation temperature and the mean mixture fraction along the center line. In Fig. 1, it can be seen that the location of the maximum is well predicted, although, the value is overpredicted by $200 \mathrm{~K}$. It is possible to see the faster increase in the predictions, compare to the experiments. Figure 1 also shows that the temperature fluctuations are in very good agreement with the experiment.

In Fig. 2 the mixture fraction is, in general, well predicted, especially after $x / D=30$. At this position the mean value is over predicted but further downstream the simulation follows the trend. Near the nozzle the predictions do not follow the concavity of the experiments, this can be attributed to the low density mesh in the near field. Nevertheless, the general behaviour is captured. It is important to note the importance of the mixture fraction prediction in CSE since all the species are derived from it.

Figure 3 shows the radial variation of the mean temperature and RMS at $x / D=15$ and 45 . At $x / D=15$, the maximum mean value is well predicted, but the flame appears to be less spread than it should be, because the maximum is located closer to the center line that the experimental maximum, this may be caused by low cell resolution at the near field. The RMS simulation captures the general trend, it presents the two maximum values, but they are not located at the same relative position corresponding to the mean temperature shown by the measurements. The second maximum is shifted by $0.5 r / D$ outwards the centerline, showing that in the simulation the flame is more spread than in the experiments. At $x / D=45$, clearly there is an overprediction of about $200 \mathrm{~K}$ of the mean temperature over the radio. The decrease of the mean temperature along the radius follows the trend of the experiments. The RMS is well predicted especially for $r / D>4$. The RMS maximum value is slightly over predicted and it is located towards the centreline respecting the measurements. The constant over prediction along the radio might be attributed to the use of steady laminar flamelet, which can not capture eventual extinction events. It is expected that at this location of the flame some extinction and premixing can occur.

Figure 4 shows the predictions of the mean mixture fraction at planes $x / D=15$ and 45 . The comparison with the experimental data shows reasonably good agreement, although at the position $x / D=15$ the values seem to be underpredicted at $Z=0.3$. This latter recalls some problem in the near filed of the nozzle or a dynamic calculations of the $S c_{t}$ number is probably needed.

\section{Pool Fire}

\section{Scenario Studied}

The configuration used for the pool fire is given in [8] where the average and variance velocities in a one meter pool fire is studied by PIV in a plane $(1 \mathrm{~m} \mathrm{x} 1 \mathrm{~m})$ at the base of the pool aligned with the central axis. The flow rate of methane is $0.066 \mathrm{~kg} / \mathrm{s} \mathrm{m} \mathrm{m}^{2}$. A flat velocity profile of $0.1 \mathrm{~m} / \mathrm{s}$ was imposed as inlet boundary condition. 


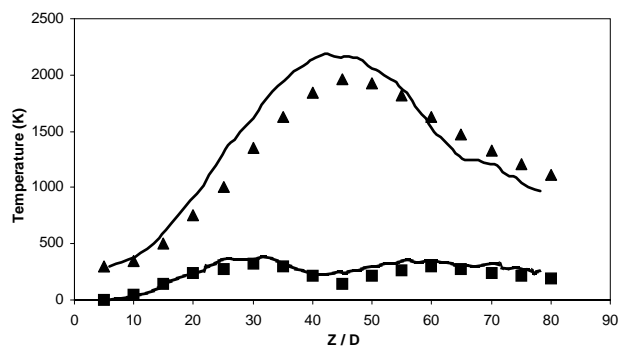

Fig. 1. Mean and RMS of temperature along the centerline $(-$ : simulation; symbols: experiments; $\boldsymbol{\Delta}$ : experimental mean value, $\mathbf{m}$ : experimental

RMS values).

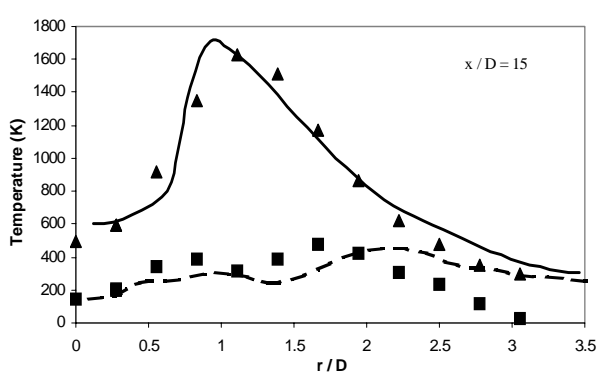

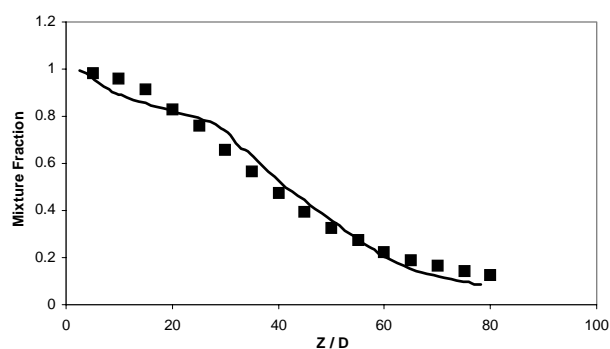

Fig. 2. Mean mixture fraction along the centerline (-: simulation, $\mathbf{m}$ : exp.).

Fig. 3. Radial distribution of mean and RMS of temperature at $x / D=15$ and 45 ( - : simulation, $\boldsymbol{\Delta}$ : exp. mean values, $\mathbf{~ : ~ e x p . ~ R M S ~ v a l u e s ) . ~}$
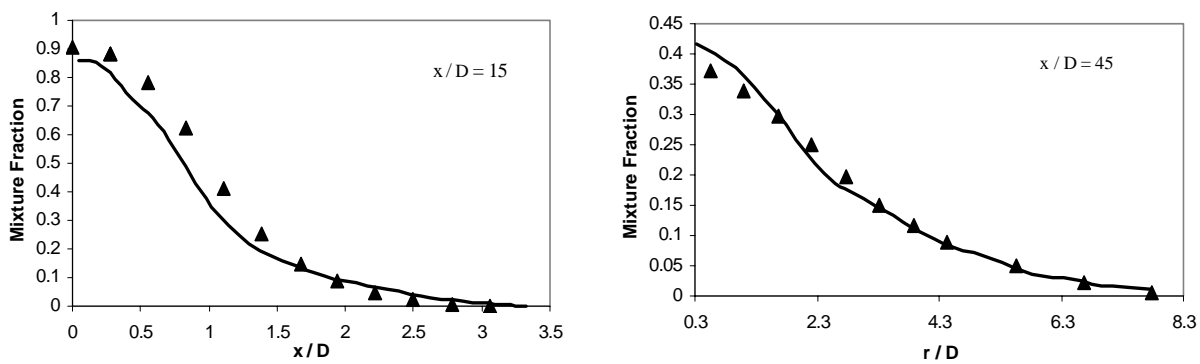

Fig. 4. Radial distribution of mean of mixture fraction at $x / D=15$ and 45. (-: simulation, $\boldsymbol{\Delta}$ : exp.).

\section{Numerical Scheme}

The numerical scheme used for the pool fire is the same used for the diffusion flame. The flamelets were computed for 15 values of dissipation rates. The minimum value being $0.2 \mathrm{~s}^{-1}$ and the maximum $30 \mathrm{~s}^{-1}$. The species boundary condition are $Y_{\mathrm{CH} 4}=1.0, Y_{\mathrm{O} 2}=0.0$ and $T=300 \mathrm{~K}$ for $Z=1$ and $Y_{C H 4}=0, Y_{O 2}=0.22$ and $T=300 \mathrm{~K}$ for $Z=0$. The mesh used in the mixture fraction space is 75 nodes more which are densely distributed close to the stoichometric mixture. 
Rectangular cells were used. The mesh used is $(90 \times 90 \times 200)$ and the domain is $(2 \times 2 \times 6) \mathrm{m}$ in $X, Y$, and $Z$ direction respectively. The lower section of the pool fire is of interest, particularly on the radiation heat flux on the pool base surface, the soot generation, the temperature and velocity profile at the bottom of fire plume.

In the present study the Radiative Transport Equation (RTE) with non-scattering gas is solved in order to obtain the radiation intensity. For simplicity we consider only one band. For the calculation of the gray or band mean absorption coefficients, $k_{n}$, a narrow band model is used [18]. The radiative transport is solved by the finite volume method [19] and [20]. The mean absorption coefficient is calculated considering the soot mass fraction and concentrations of $\mathrm{CH}_{4}, \mathrm{O}_{2}, \mathrm{CO}_{2}, \mathrm{H}_{2} \mathrm{O}, \mathrm{CO}$ and $\mathrm{N}_{2}$.

The Moss soot model [21] is employed. This model uses the soot particle number density and the mass density to describe the soot formation. The model includes inception, coagulation, growth and oxidation processes. Two extra transport equations are solved for soot density and concentration. A more detailed discussion regarding the concepts behind the soot model can be found in [22].

\section{Results for Pool Fire}

In this section the results for the pool fire are presented for the mean velocity, temperature, radiative heat flux and soot concentration.

Figure 5 shows the mean velocity along the centre line. In comparison with Mc Caffrey's correlation the predictions show reasonable good agreement, although clearly over predicts by $15 \%$ the velocity from $Z / Q^{1 / 4}=0.11$ onwards.

Figure 6 shows the mean velocity variations with radio and compared with experimental data of Tieszen \& O'Hern [8]. The centre velocity is reasonably predicted along the centre line. At $Z / D=0.25$ the calculated velocities increase towards the outer section of the flame and are close to the experiments until $r / D=0.2$, where there is an overprediction of the velocity at $Z / D=0.3$. In the external region there exists a constant overestimation of the vertical velocity.

For $Z / D=0.5$ the pattern is similar to $Z / D=0.25$, i.e., a good estimation of the central velocity and an overprediction of both the location and the value of the maximum velocity. Approximately the same can be seen at $Z / D=0.9$ where the central velocity overprediction becomes more important and the experiments do not show a peak of velocity while the simulation predicts one at $r / D=0.1$.

Figure 7 shows the soot concentration along the centreline. In general the simulation predicts values of one order of magnitude smaller than the results obtained by Rawat et al. [23]. At $Z / D=3.5$ is the maximum soot concentration. It can be seen further downstream that the soot is oxidised as stated by Rawat et al. [23]. The oxidation is mainly due to the $\mathrm{O}_{2}$ rather than $\mathrm{OH}$. It is important to point out that the under prediction of the soot may lead to an over prediction of temperature and consequently of velocity due to the buoyant forces. 


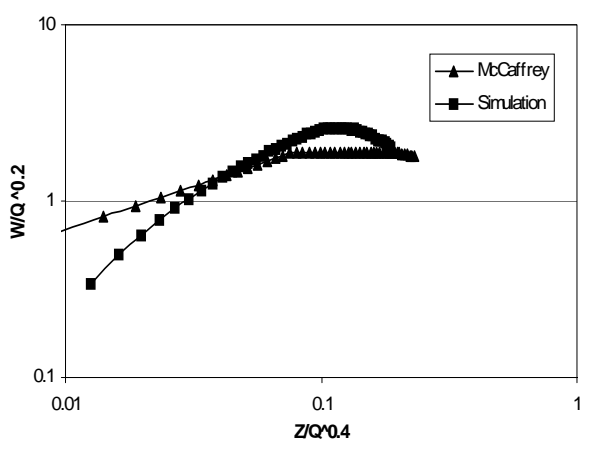

Fig. 5. Mean velocity at centerline.

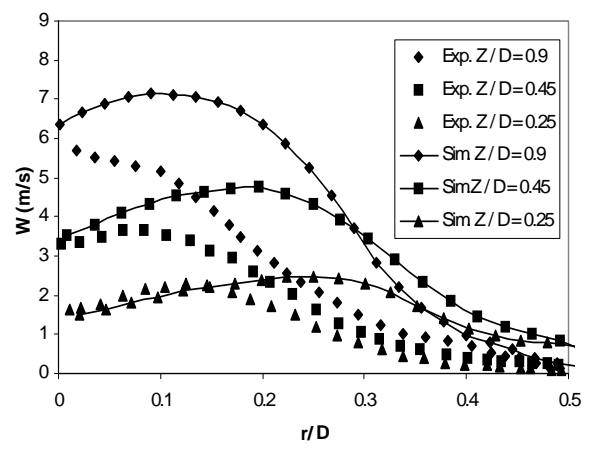

Fig. 6. Mean velocity radial distribution at $\mathrm{Z} / \mathrm{D}=0.25,0.45$ and 0.9 , respectively.

In Fig. 8 the soot concentration on planes $Z / D=0.5,1.0$ and 1.5 is shown. The soot peak is shifted towards the centre. This is mainly attributed to the soot growth due to $Y_{\mathrm{C} 2 \mathrm{H} 2}$ and temperature, which follow the same pattern. Moreover, considering the maximum temperature remains almost constant on different planes (see Fig. 11) and $Y_{\mathrm{C} 2 \mathrm{H} 2}$ does not change significantly, as expected. At $Z / D=2.5$, the production of soot increases reaching the tip of the flame, in the intermittent zone.

In Figs. 7 and 9 the maximum soot concentration and temperature do not occur at the same location. This is pointed out by Rawat et al. [23].

The mean temperature and the predictions obtained by Rawat et al. [23] at different planes are shown in Fig.10. There is high temperature region at the stoichometric mixture which corresponds with the maximum velocity. The evolution of the temperature profile on different planes downstream is characterised by a temperature peak at stoichometric mixture with a minimum at the centre. At $Z / D=0.06$ both simulations locate the maximum temperature about $r / D=0.4$, although ULFM over predicts CSE by $50 \%$. At $Z / D=2.5$ and 3.0 CSE does not predict a low temperature at the centre, while ULFM shows this pattern at $Z / D=3.0$, indicating that ULFM tends to predicts larger buoyancy forces that CSE.

As the large eddies evolve downstream, the mixing becomes important and the temperature is homogenised by the turbulence.

The mean heat flux due to radiation is shown Fig. 11. The same behavior as in the case of temperature and velocity fields can be observed. Note the maximum soot peak in Fig. 8 correlates with the maximum radiation heat flux in Fig. 11 for planes $Z / D=1.0$ and 1.5 , although, this is not the case at $Z / D=2.5$.

However the simulation captures the puffing nature of the fire, can be concluded from the results that not enough turbulence is generated by eddies at the base of the pool. These eddies are responsible for mixing the flow downstream. This might be occasioned either by a coarse mesh or a deficient turbulent sub-grid model. 


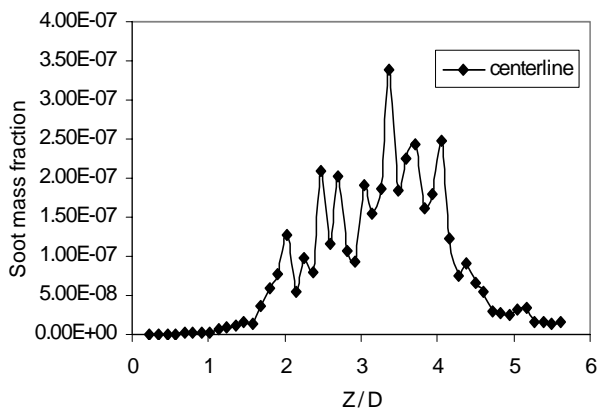

Fig. 7. Soot mass concentration at concentration at center line.

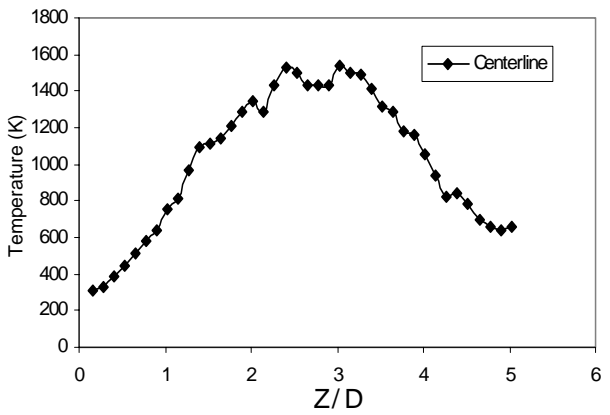

Fig. 9. Mean temperature at centerline.

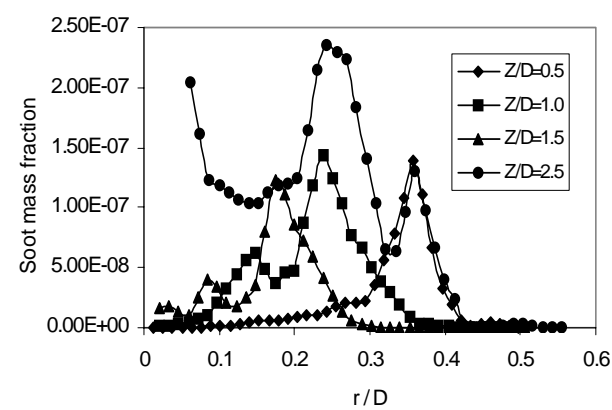

Fig. 8. Soot mass concentration for planes $Z / D=0.5,1.0,1.5$ and

2.5 at the centre plane.

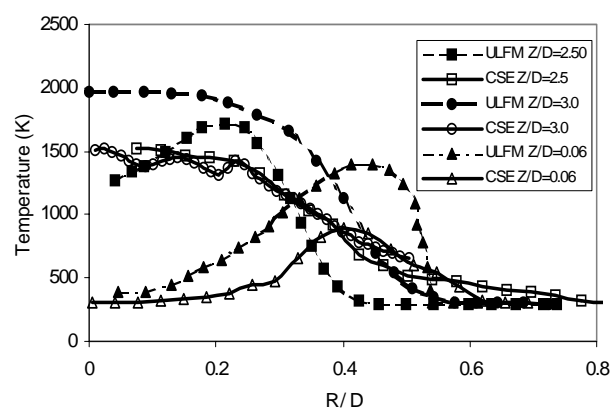

Fig. 10. Mean temperature at different planes.

Vortices generated at the pool rim do not develop far enough towards the pool centre. The mechanism of air entertainment is underpredicted. Consequently, there is not enough mixing close to the pool and the up-going neck formed at the pool centre is wider than in the experiments. A consequence of this is that the stoichometic mixture, close to the base, is located in the periphery of the pool and the heat is released at the pool edge producing an increase of velocity at the periphery of the plume. This latter phenomenon is not observed in the experiments, where large vortices are observed entraining from the sides of the pool towards the centre, producing more mixing.

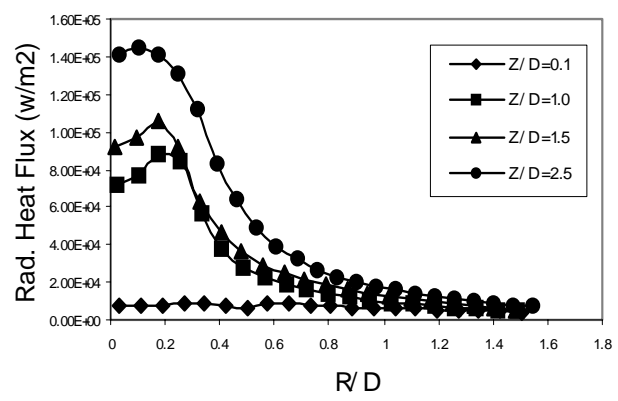

Fig. 11. Radiation fluxes at different planes. 
In Fig. 6 the total predicted momentum is larger that in the experiments. This may be attributed to several factors. The mesh size can influence in the spread rate. Another important point is the correct prediction of the soot, which directly affects the radiation flux and the temperature, resulting in an overprediction of vertical momentum. Without the correct behavior of large eddies at the base, an important sink of energy is lost which could lead to overprediction of the momentum further downstream. This same tendency is observed in [23].

\section{CONCLUSIONS AND FUTURE WORK}

A modified version of the Conditional Source Term (CSE) model has been developed in this work. The new approach was applied to a diffusion flame (Flame D) and to a $1 \mathrm{~m}$ diameter pool fire. In both cases the results reasonably agree with the experiments.

The main objective of this paper was to prove the possibility of CSE as a model of relatively low computational cost to be applied in large fires. In the case of the flame D the results were obtained in $10 \mathrm{hrs}$ and for the pool fire in $24 \mathrm{hrs}$ on a $3.06 \mathrm{GHz}$ processor with the meshes considered above. About $70 \%$ of the CPU time, in the pool fire simulation was dedicated to the radiation model for the pool fire.

Some issues remain to be addressed in the combustion model, such as, the number of functions considered in the inversion and $\lambda$ (Eq. 3). Another important aspect is the consequences of negative coefficients; in this work they were normalized avoiding negative values, but their meaning is not clear. The inversion used could be improved using a starting guess for the conditional; this in turn could be provided either by the previous time solution or by the upstream conditional solution. This latter option could be an interesting alternative because it would introduce a Lagrangian concept to the model.

It is clear that is not possible to predict events such as extinction and ignition using a steady laminar flamelet. Therefore, regions of the flame where these events can take place will not be accurately predicted. Nevertheless, if extinction events could be introduced into the basis this problem could be overcome.

\section{ACKNOWLEDGMENTS}

The calculation of the Steady State Flamelets were carried out using the Flame Master code by Pitsch, $\mathrm{H}$. This work has been developed inside the EC Framework 5.

\section{REFERENCES}

[1] Cook, A.W., and Riley, J.J., "A Subgrid Model for Equilibrium Chemistry in Turbulent Flows,” Phys. Fluids, 6, p. 2868, 1994.

[2] Pierce, C.D., and Moin, P., "A Dynamic Model for Subgrid-scale Variance and Dissipation Rate of a Conserved Scalar,” Physics of Fluids, 10, Iss. 12, (1998).

[3] Bushe, W.K., and Steiner, H., "Conditional Moment Closure for Large Eddy Simulation of Nonpremixed TurbulentReaction Flows," Physics of Fluids, 11 Iss. 7, (1999).

[4] Bushe, W.K., and Steiner, H., "Laminar Flamelet Decomposition for Conditional Source-term Estimation,” Physics of Fluids, 15, Iss. 6, (2003). 
[5] Cook, A.W., Riley, J.J., and Kosály, G., "A Laminar Flamelet Approach to Subgrid-scale Chemistry in Turbulent Flows,” Combust. Flame, 109, pp. 332341, (1997).

[6] Pitsch, H., and Steiner, H., "Large-eddy Simulation of a Turbulent Piloted Methane/air Diffusion Flame (Sandia flame D),” Physics of Fluids, 12 Iss. 10, (2000).

[7] Barlow, R.S., and Frank, J.H., "Piloted $\mathrm{CH}_{4} /$ Air Flames C, D, E, and F Release 2.0, 20-JAN-2003," TNF Workshop, Sandia National Laboratories, 1998, http://www.ca.sandia.gov/TNF/DataArch/FlameD.html.

[8] Tieszen, S.R., and O'Hern, T.J., "Experimental Study of the Flow field in and Around a One Meter Diameter Methane Fire," Combustion and Flame, 129, pp. 378-391, (2002).

[9] Bilger, R.W., and Klimenko, A.Y., "Conditional Moment Closure for Turbulent Combustion,” Progress in Energy and Combustion Science, 25, pp. 595-687, (1999).

[10] Craig, I.J.D., and Brown, J.C., Inverse problems in astronomy, A Guide to Inversion Strategies for Remotely Sensed Data, Adam Hilger Ltd, 1986.

[11] Grout, R., and Bushe, W.K., "Analysis of the Sandia Flame D Using an Implementation of Conditional Source-term Estimation in a Commercial RANS Solver," Dept. of Mechanical Engineering, University of British Columbia, Vancouver, Personal Communication.

[12] Peters, N., "Laminar Diffusion Flamelet Models in Non-Premixed Turbulent Combustion," Prog. Energy Combust., 10, p. 319-339, (1984).

[13] Pitsch, H., Seiser, R. and Varathajan, B., FlameMaster Computer Code, 1996.

[14] Peters, N., and Rogg, B., Reduced Kinetic Mechanism for Applications in Combustion Systems, Springer, 1993.

[15] Twomey, S., "Introduction to the Mathematics of Inversion in Remote Sensing and Indirect Measurements,” Developments in Geomathematics, Amsterdam, Elsevier scientific publishing company, 1977, p. 3.

[16] Girimaji, S., and Zhou, Y., "Analysis and Modeling of Subgrid Scalar Mixing Using Numerical Data,” Physic of Fluids, 8, p. 5, (1996).

[17] Barlow, R.S., and Frank, J.H., "Effect of Turbulence on Species Mass Fractions in Methane/Jet Flames,” Proc. Combust. Inst., 27, p. 1087, (1998).

[18] Grosshandler, W., "A Narrow Band Model for Radiation Calculations in a Combustion Enviroment,” 1993, NIST, Gaithersburg, Maryland, 20899.

[19] Raithby, G.D., and Chui, E.H., "A Finite Volume Method for Predicting Radient Heat Transfer in Enclosures with Participating Media," Journal of Heat Transfer, 112, Iss. 2, p. 415-423, (1990).

[20] McGrattan, K.B., "Fire Dynamics Simulator - Technical Reference Guide, 2002,” NIST. 
[21] Brookes, S.J., and Moss, J.B., "Prediction of Soot and Thermal Radiation Properties in Confined Turbulent Diffusion Flames," Combust. Flame, 116 (4), pp. 486-503, 1999.

[22] Wen, Z., Yun, S., Thomson, M.J., and Lightstone, M.F., "Modeling Soot Formation in Turbulent Kerosene/air Jet Diffusion Flame," Combustion and Flame, 135, p. 323-340, (2003).

[23] Kronenburg, A., Bilger, R.W., and Kent, J.H., "Modeling Soot Formation in Turbulent Methane-air Jet Diffusion Flames," Combustion and Flame, 121, pp. 24-40, (2000).

[24] Said, R., Garo, A., and Borghi, R., "Soot Formation Modeling for Turbulent Flames,” Combustion and Flame, 108, pp. 71-86, (1997).

[25] Rawat, R., Pitsch, H., and Ripoll, J.F., "Large-eddy Simulation of Pool Fires with Detailed Chemistry Using an Unsteady Flamelet Model," in Proceeding of the Summer Program 2002, Center for Turbulence Research, 2002. 